\title{
An Improved Immune Genetic Algorithm for Capacitated Vehicle Routing Problem
}

\author{
Cheng LinHui ${ }^{1,2, *}$ and Zhong Luo ${ }^{2}$ \\ ${ }^{I}$ College of Computer Science, South-Central University for Nationalities, Wuhan 430074, China; ${ }^{2}$ College of Computer \\ Science and Technology, Wuhan University of Technology, Wuhan 430070, China
}

\begin{abstract}
The capacitated vehicle routing problem is an NP-hard problem. In this paper, it proposes a kind of dynamically adaptive immune genetic algorithm with automatic immune monitoring function. By setting an immune monitor, the algorithm automatically obtains the timing of regulating the antibody concentration and vaccination, and the dynamic antibody concentration regulation mechanism is designed to keep the variety of the antibody evolution process and prevent the algorithm from getting in trouble with local optimal solution. What is more, the manner of vaccination and extraction is designed to improve the global optimization of the algorithm, and the elite antibodies ensure the convergence. The experiment results show that the improved immune genetic algorithm takes good performance on the capacitated vehicle routing problem.
\end{abstract}

Keywords: Immune genetic algorithm, rank method, vehicle routing problem.

\section{INTRODUCTION}

The vehicle routing problem is proposed (VRP) in 1959 by the famous scholar Dantzig [1], and this problem is a hot issue concerned by operations research experts and other scholars, which is an important supporting technology for efficient distribution of transportation. The Capacitated Vehicle Routing Problem (CVRP) is a constraint model of VRP problem, which can be described as follows: For a number of customer points and some capacitated vehicle, the problem becomes how to arrange a reasonable vehicle route to ensure that the vehicle can be go through the customer points orderly with shortest routine distance and minimized cost, etc.

CVRP is a typical combinatorial optimization problem, research on CVRP is of important theoretical significance and practical value. But CVRP is a kind of NP-hard problem, it means when problem scale is large, optimal solution can not be solved by using mathematical methods or traditional heuristic algorithms. In recent years, many researchers have proposed a variety of swarm intelligence optimization algorithms to solve CVRP problem, such as the Genetic Algorithm (GA) borrowed from survival of the fittest mechanism $[2,3]$, Immune Algorithm(IA) inspired by biological immune system $[4,5]$, and the ant colony algorithm from simulating ant colony foraging behavior [6, 7], etc. These algorithms are simulating social behavior and physiology of the human or animal which provides a new approach and new methods for solving complicated CVRP. However, it is not satisfying enough by simply taking single algorithm, so many domestic and foreign researchers to make integration of intelligent optimization algorithms, and design a more effective method to solve this problem $[8,9]$.
Immune Genetic Algorithm (IGA) is a novel fusion algorithm which is an improved genetic algorithm based on biological immune mechanism. It integrated adaptive recognition, self-learning, immune memory, pattern recognition into the genetic algorithm, which to some extent inhibited individual's degradation of the genetic algorithm's completely random optimization process and fluctuation in the late evolution. The algorithm can improve the overall performance and convergence, but it is still a genetic algorithm to some extent and difficult to get rid of the defect about premature convergence.

Therefore, this article proposes a dynamic adaptive immune genetic algorithm to increase antibody diversity and improve the antibody quality. By setting the immune alarm, the algorithm could dynamically adjust the antibody concentration and operate vaccination timely. We design antibody concentration suppression method based on rank mechanism to reduce the probability of antibody's premature convergence, design vaccines extraction and vaccination methods, according to the characteristics of the problem, to improve the performance of the global search algorithm. To test the performance of the algorithm, it is applied to multiple test cases of CVRP to do simulation experiments and results analysis.

\section{CVRP PROBLEM MODEL}

The requirement of CVRP is that any vehicle's cargo on the traveling path is not allowed to exceed the maximum capacity limit of the vehicle. Supposing that capacity constraint for all vehicles is equal, and the some other requirement must be met: each customer can only be visited once by a vehicle and the customer's demand must be met; all vehicles depart from a single distribution center, and vehicles are required back to the starting point. The mathematical model of CVRP established as below. 
Given a distribution center (distribution center is represented by 0$)$ and $n$ customer points $(1,2, \ldots)$, each customer demand is set as $d_{i}(i=1,2, \ldots n)$, and the distance from customers point $i$ to point $j$ is $R_{i j}(i, j=1,2, \ldots n)$, each vehicle's cargo capacity is $\mathrm{C}$. Set $M$ as the number of actually used vehicles, and set the total number of customer points passed by the vehicle number $k(k=1,2, \ldots M)$ as $b_{k}\left(b_{k}=1,2, \ldots n\right)$. The set $V_{k}=\left\{v_{k i} \mid 0 \leq i \leq b_{k}\right\}$ is to represent each customer points passed by the vehicle of number $k$, in which $v_{k i}$ is the custom point $i$ served by vehicle $k$. Thus, the CVRP model is

$$
\begin{aligned}
& \quad \min f=\sum_{k=1}^{M}\left(\sum_{i=1}^{b_{k}} R v_{k(i-1)} v_{k i}+R v_{k 0} v_{k b_{k}}\right) \\
& \text { s.t. } \sum_{i=1}^{b_{k}} d_{v_{k i}} \leq \mathrm{C}, k=1,2, \ldots M \\
& 1 \leq b_{k} \leq \mathrm{n}, k=1,2, \ldots M \\
& \sum_{k} b_{k}=\mathrm{n}, k=1,2, \ldots M \\
& V_{k}=\left\{v_{k i} \mid v_{k i} \in\{1,2, \ldots, n\}, 0 \leq i \leq b_{k}\right\} \\
& V_{k 1} \cap V_{k 2}=\Phi, \forall k 1 \neq k 2
\end{aligned}
$$

The objective function $f$ is the solution of the problem, i.e., the shortest path length. Constraint (1) is the capacity limitations of the vehicle, which is to ensure that the total demand for each customer point on each sub-path must be less than the vehicle's maximum cargo capacity, constraints (2) ensures that the customer points on each path does not exceed the total customer number of points, the constraints (3) (4) (5) ensure that every customer point would occupy only one vehicle, and define each customer point can only be visited by single vehicle for one time.

\section{THE IMPROVED IMMUNE GENETIC ALGO- RITHM FOR CVRP}

\subsection{Theory of IGA}

The immune system is composed of antigen recognition system, memory mechanism, and antibody promotion and suppression section. In immune genetic algorithm, antibody population's evolutionary learning process and antigen recognition are the optimization process. The antigen is corresponding to the objective function, and the antibody is corresponding to the feasible candidate solutions. The matching degree of candidate solutions to the objective function reflects the affinity between antigen and antibody, and the higher the matching degree indicates the greater affinity and the better the quality of candidate solutions. The affinity of antibodies is reflected in the similarity degree candidate solutions, the assessment of antibody group is undertaken by the two affinities. Through crossover, mutation genetic manipulation and clonally selection operation, the antibody groups are updated, and the immune memory mechanism record each generation antibody group's optimal antibody group. Through mutual promotion and inhibition between antibodies, search efficiency is improved near optimal solutions to achieve the goal of convergence to the global optimum.

\subsection{Improvement Strategies}

(1) Setting immune monitor

The immune monitor decides whether to perform antibody concentration inhibition antibody operation and vaccination operation by calculating the optimal antibody invariant algebra. When the optimal antibodies do not update for many generations, it indicates that the superior group antibody populations is accumulating in the evolutionary process, the concentration of similar antibodies is increasing, and antibody is going into local optimum risk. At this time, the monitor gives the warning algorithm should start the concentration of antibody inhibition operation, increasing antibody diversity and preventing antibody population from converging to local optimum. At the same time, the vaccination operation is started in order to improve the genetic quality of the new antibody population, accelerating global optimization efficiency.

(2) Dynamic concentration inhibition operation of antibody

This paper presents a dynamic concentration inhibition operation of antibody based on the rank method, the method is proposed by Fonseca and Fleming in 1998 when seeking multi-objective optimization problem Pareto solution [10], and the purpose is to increase the diversity of population.

First, the antibody population is divided into several subgroups according to the affinity level between the antibody and the antigen, and set the current scale of antibody group $A$ as $X(A)$, in which there are $Y$ different affinity levels. All antibodies of affinity $M\left(A_{i}\right)(i=1,2, \ldots, Y)$ form a sub-group $A_{i}(i=1,2, \ldots, Y)$, each antibody sub-group and affinity $M\left(A_{i}\right)$ are taken as the respective sub-groups ranking standard. And according to the general ranking method shown in formula (6), calculate the rank values $R\left(A_{i}, A\right)$ of the respective sub-groups. Then determine the maximum size $X\left(A_{i}\right)$ of each sub-group, according to the size of the group $X(A)$, the number of sub-groups $Y$ and the rank $R\left(A_{i}\right)$ of each sub-antibody population. If the antibodies' number of a subgroup exceeds its' maximum size, some old antibodies, whose number is over maximum size, will be replaced by the new antibodies random generated.

$$
\begin{aligned}
\mathrm{R}\left(A_{\mathrm{i}}, A\right) & =\sum_{\substack{j=1 \\
j \neq i}}^{Y} t_{j} \mathrm{i} \\
\text { s.t. } \quad t_{j} & =\left\{\begin{array}{ll}
1 & \text { if } M\left(A_{i}\right)<M\left(A_{j}\right) \\
0 & \text { else }
\end{array}(i=1,2, \ldots, Y)\right.
\end{aligned}
$$


$X\left(A_{i}\right)=\frac{X(A)}{Y+1-R\left(A_{i}, A\right)}(\mathrm{i}=1,2, \ldots, \mathrm{Y})$

From formulas (7), the algorithm makes different degrees of restrictions on the optimum antibodies and poor antibodies, thus avoiding premature convergence by optimum antibodies accumulating in the evolution of the antibody, strengthening antibody poor chance of survival, increasing antibody population diversity, improving the search range, and accelerating the solving process. The allowed size of sub-group antibody in operator $X\left(A_{i}\right)$ is decided by the size of antibody groups, the number of sub-antibody groups $Y$ as well as the ranking value of sub-group $R\left(A_{i}, A\right)$, which means that if the size of the sub-antibody groups are adjusted, the adjusted results are not same, but changes with these three values' dynamic change, which shows the adaptability of this algorithm.

\section{(3) Vaccine extraction method}

According to the characteristics of the integers sequence coding in CVRP problem, the optimal antibody genes in different groups of the memory were compared. Set the precursor code probability table and the subsequent coding probability table to each gene, and calculate them to find a stable precursor gene and subsequent gene fragment, if these gene fragments can be visited by one vehicle, extract vaccine from slices these genes.

\section{(4) Method of vaccination}

For extracted vaccines' fragments of vaccination needed antibody, it ensure that the fragment can be vehicle visited, the adjustment of the rest position of the antibody gene makes every customer of gene sequence numbered only once.

\section{(5) Immune memory}

Using elitist strategy, after every antibody generation update, the best optimum antibodies and some better optimum antibodies are put into memory. By the use of this memory, it achieves the search process speed up and search ability improved.

\subsection{Code Design}

(1) Antibody encoding and decoding

For VRP problem, we use integer sequence antibody encoding method, for example, those containing 9 customer points (distribution centers numbered 0 ) code is: $9 \rightarrow 7 \rightarrow 8$ $\rightarrow 2 \rightarrow 1 \rightarrow 4 \rightarrow 6 \rightarrow 3 \rightarrow 5$. Antibody decoding process is: checking customer number which appears orderly in antibody, if the current sub-path caused overloading by adding the next customer, then this customer is taken as the first customer of the next sub-path.

\section{(2) Crossover and mutation operator}

The partial cross-matching method means generating two genes intersection first in the chromosome length randomly, defining the area between the two points as matching area, exchanging gene string in the two paternal matching area, getting two new intermediate individual, then adjusting re- duplicate genes of the two intermediate individuals outside the region, getting two new individuals.

About the reverse mutation method, it is inverting substring randomly from a selected section of antibody.

\subsection{Algorithm Process}

The algorithm process is shown in Fig. (1), and the steps are as below:

\section{Step 1. Initialization operation}

Step 1.1 Initialize antibody size $X(A)$ and the maximum evolution algebra $T$, set the crossover probability $P c$ and mutation probability $P m$, specify the maximum immune monitoring threshold $V$;

Step 1.2 The current evolution algebra is $t=1$, current immune monitor is $v=1$.

Step 2. Randomly generate an initial antibody group $A$ $(t)$. That is an ordered sequence of $X(A)$ random set of customers point codes.

Step 3. Calculate the affinity between antibody and antigen.

Step 4. Perform immune memory operation

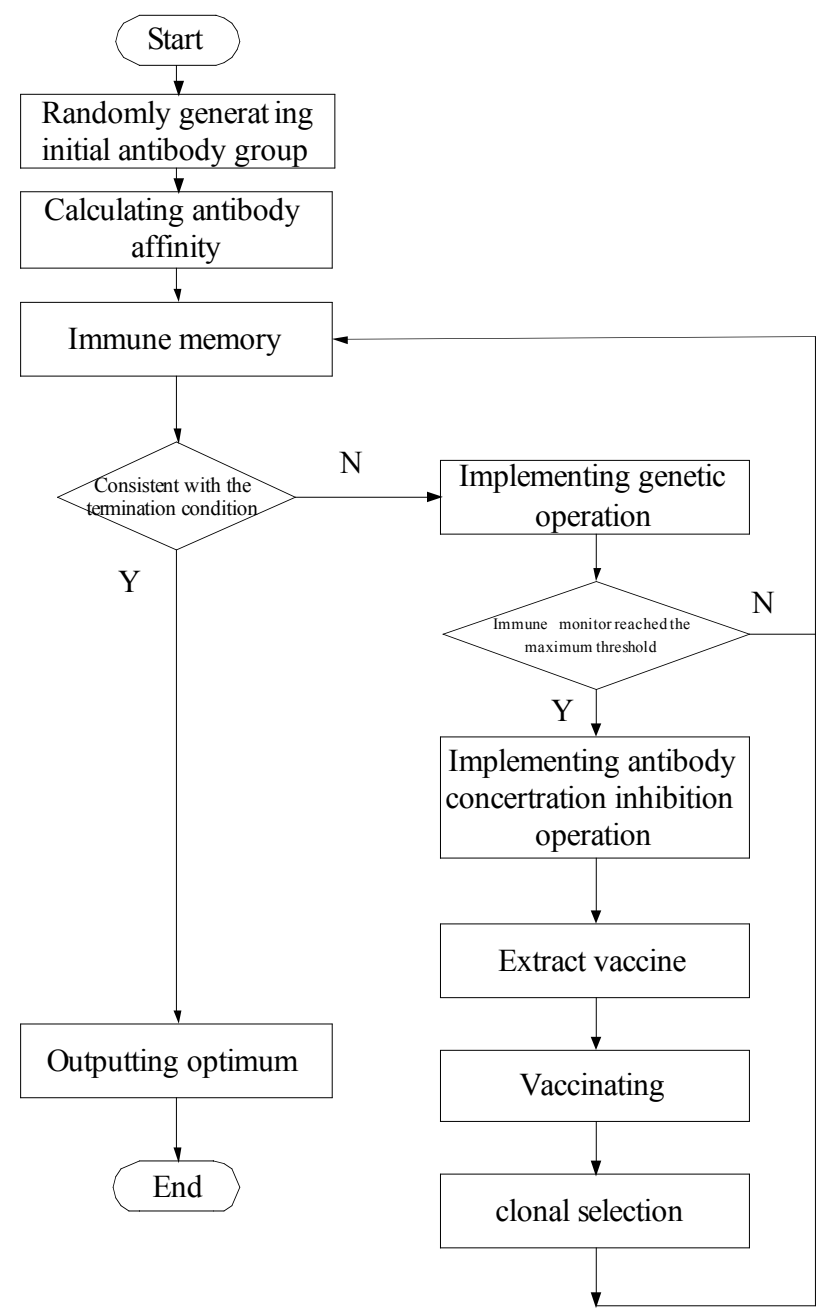

Fig. (1). Flowchart of the improved immune genetic algorithm. 
Table 1. Results of computational experiments.

\begin{tabular}{|c|c|c|c|c|c|c|c|}
\hline \multirow{2}{*}{ Test cases } & \multicolumn{3}{|c|}{ Problem Characteristics } & \multicolumn{4}{c|}{ Comparison of the Experimental Results } \\
\cline { 2 - 8 } & $\begin{array}{c}\text { Number of } \\
\text { Customers }\end{array}$ & $\begin{array}{c}\text { Number of } \\
\text { Vehicles }\end{array}$ & $\begin{array}{c}\text { Vehicle } \\
\text { Capacity }\end{array}$ & $\begin{array}{c}\text { Current } \\
\text { Optimum }\end{array}$ & Osman's SA & $\begin{array}{c}\text { Algorithm in } \\
\text { this Paper }\end{array}$ \\
\hline \hline E-n22-k4 & 21 & 4 & 600 & 375 & 375 & 375 & 375 \\
\hline E-n30-k3** & 29 & 3 & 4500 & 569 & 569 & 569 & 569 \\
\hline E-n50-k5 & 49 & 5 & 160 & 521 & 528 & 524.81 & 524.61 \\
\hline E-n100-k8 & 99 & 8 & 200 & 815 & 826.14 & 839.73 & 826.14 \\
\hline
\end{tabular}

Step 4.1 Save the current optimal antibodies $A_{\text {best }}$ and some other better antibodies to memory;

Step 4.2 If $t>1$, the current optimal antibody has not been updated, the immune monitor $v=v+1$.

Step 5. Determine whether the algorithm termination condition is satisfied, i.e., $t<T$, if not, then continue step 6 , otherwise, the algorithm stops and outputs optimal solution.

Step 6. Genetic operation

Step 6.1 Perform crossover operation based on the set crossover probability of antibody group, get $A c(t)$;

Step 6.2 Perform mutation of antibodies based on mutation probability, and get $A m(t)$

Step 7. Calculate $v$, i.e. the immune monitor's value, if $v>$ $V$, continue Step 8; otherwise turn to Step 4.

Step 8. Perform the concentration of antibody inhibition mechanism based on rank methods

Step 8.1 Calculate affinity of each antibody population of antibody to the antigen;

Step 8.2 Calculate the affinity level of the antibody;

Step 8.3 According to the affinity level, divide antibody group into several sub-antibody groups;

Step 8.4 Calculate $X\left(A_{i}\right)$, i.e., the largest size of each antibody sub-group;

Step 8.5 Analyze whether $N\left(A_{i}\right)$ (the antibody number of each antibody sub-group) exceeds $X\left(A_{i}\right)$ (the maximum size of each antibody sub-group), if it is, then randomly generate $X\left(A_{i}\right)-N\left(A_{i}\right)$ antibodies of different affinities to replace them, if not, continue Step 9.

Step 9. Extract vaccine.

Step 10. Vaccinate the weak antibody.

Step 11. Perform antibody clonally selection operation on the basis of affinity strength, the greater affinity antibodies will get more chances for clone. Get $A(t+1), t=t+1$, turn to Step 4 .

\section{EXPERIMENT AND ANALYSIS}

In order to verify the feasibility and effectiveness of the algorithm, experimental program is written in $\mathrm{VC}++$ environment, and select "Christofides \& Eilon" a number of different sizes CVRP problem in common standard test case library [11] as an example of test, in the algorithm execution, and set different parameters depending on the specific issues, antibody size and maximum evolution algebra are increasing with the number of customer points increasing. The general crossover probability value is 0.9 , mutation probability value is 0.1 in general, and the largest immune monitoring threshold is in the range of 10 to 100 . For each test cases it run randomly 10 to 30 times, recording the shortest path value and each sub-path information, and compare the test results with the currently known best results and some other intelligent algorithms experimental results [12]. Names of the test cases, problem characteristics, and the experimental results are shown in Table 1. The results of each test case's optimal result path are shown in Figs. (2-5).

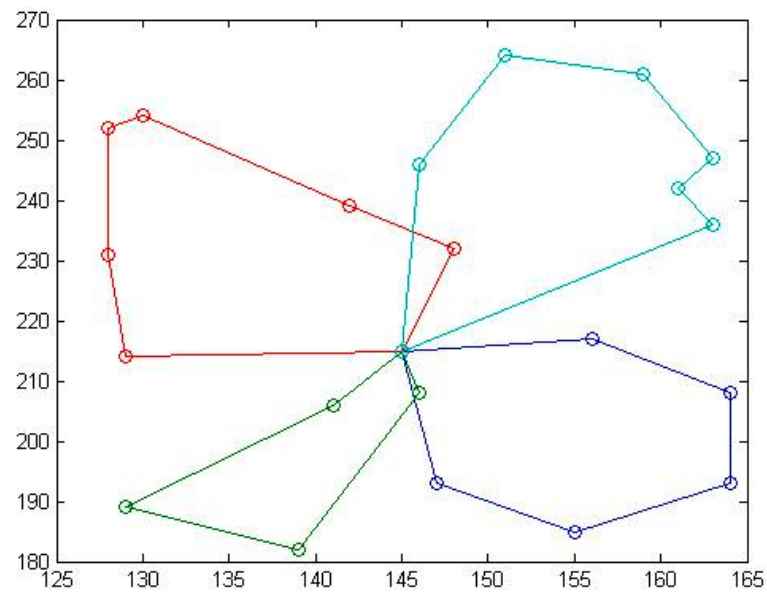

Fig. (2). E-n22-k4 optimal path diagram.

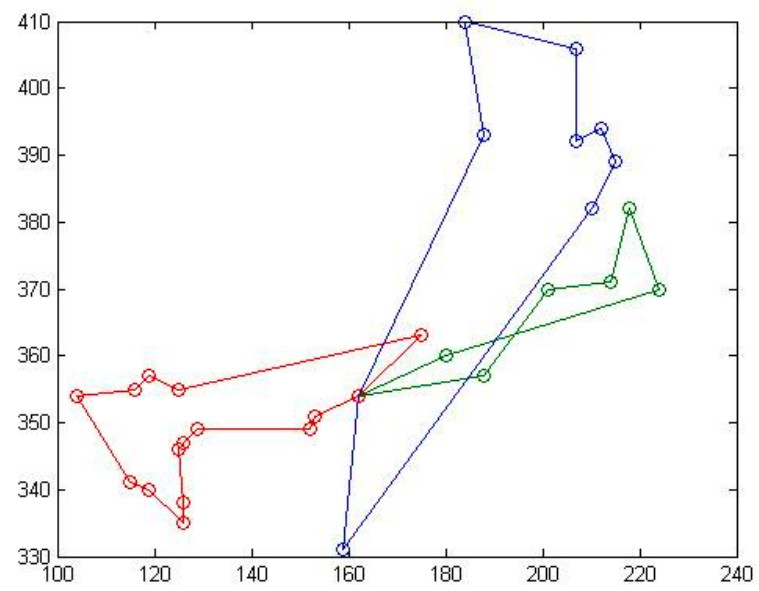

Fig. (3). E-n30-k3** optimal path diagram. 


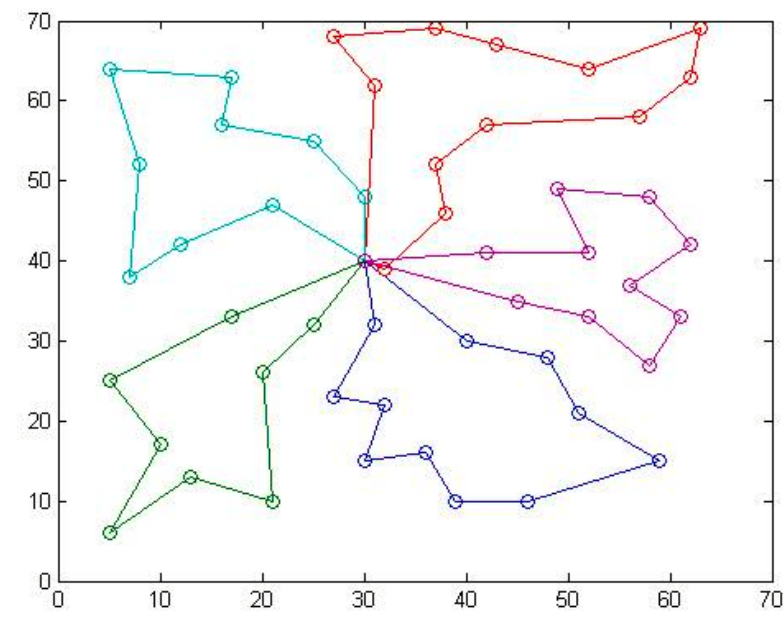

Fig. (4). E-n50-k5 optimal path diagram.

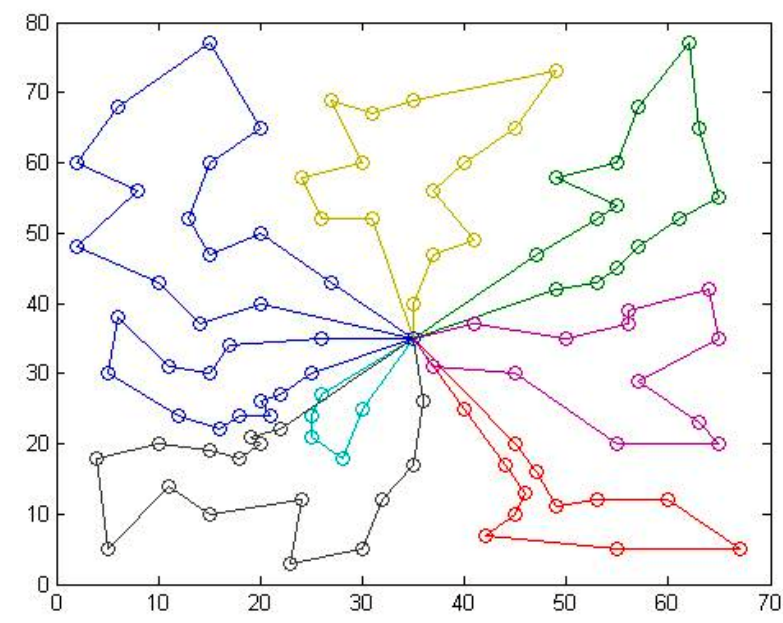

Fig. (5). E-n100-k8 optimal path diagram.

From Table 1, the best solution in the experiment is the case that the customer number is less than 50 points, and the algorithms we design in the paper can obtain currently known optimal solutions, which shows that the algorithm is feasible and effective.

For test cases with many customers' points, the algorithm of this paper is not able to get the optimal solution so far, but the experimental results obtained are very close to the current value of the best known. For example, the test case E- $\mathrm{n} 50-\mathrm{k} 5$ has 50 customer $\mathrm{s}$, whose result is only with $0.007 \%$ relative deviation close to the current optimal solution, and the test case with 100 customer point E-n100-k8's result are only with $0.013 \%$ relative deviation close to the current optimal solution. However, compared with Osman's SA algorithm and GA algorithm, the proposed algorithm is the best one for E-n50-k5 and equal to Osman's SA while better than GA for E-n100-k8, which shows that the quality improved immune genetic algorithm proposed in this paper are more competitive.

In addition, Table 2 shows the test case E-n50-k5's optimal solutions 524.61's sub-paths. It can be seen that the respective load rate of each sub-path is quite high, all vehicles' load rate are more than $93.1 \%$, the best sub-path reaches $100 \%$, therefore the proposed algorithm is effective.

\section{CONCLUSION}

Based on the study on CVRP problem and immune genetic algorithm, for the problem of algorithms, an improved immune genetic algorithm is proposed. The algorithm is to increase antibody's diversity and improve the quality of the starting antibody genes, which is designed based on dynamic antibody concentration and rank methodology adjusting mechanism, and through dynamic vaccination and vaccine extraction, the quality is improved to ensure the diversity of algorithm in the evolutionary process and avoid the convergence to local optima. The improved algorithm is used for solving CVRP problems, and some cases are chosen randomly from the VRP problem standard test library, and experimental results show that the smaller the CVRP problem algorithms are able to find the optimal solution so far, for the large CVRP problem, the proposed algorithm is also able to get satisfactory solution, which prove the validity and global optimization capability of the improved immune genetic algorithm.

\section{CONFLICT OF INTEREST}

The authors confirm that this article content has no conflict of interest.

\section{ACKNOWLEDGEMENTS}

This work is supported by the project of National Natural Science Foundation of China (No. 61379059) and Hubei Province Natural Science Foundation (No. 2014CFB912).

Table 2. Sub-paths of test case E-n50-k5's optimal solution.

\begin{tabular}{|c|c|c|c|}
\hline Sub-Path Number & $\begin{array}{c}\text { Customer Numbers of Each } \\
\text { Sub-Path }\end{array}$ & Load Rate of Each Vehicle & Customer's Numbering Sequence of Each Sub-Path \\
\hline \hline 1 & 11 & $100 \%$ & $0-12-37-44-15-45-33-39-10-49-5-46-0$ \\
\hline 2 & 10 & $99.4 \%$ & $0-11-2-29-21-16-50-34-30-9-38-0$ \\
\hline 3 & 9 & $98.1 \%$ & $0-18-13-41-40-19-42-17-4-47-0$ \\
\hline 4 & 9 & $95 \%$ & $0-27-48-23-7-43-24-25-14-6-0$ \\
\hline 5 & 11 & $93.1 \%$ & $0-8-26-31-28-3-36-35-20-22-1-32-0$ \\
\hline
\end{tabular}




\section{REFERENCES}

[1] G.B. Dantzig and J.H. Ramser, "The truck dispatching problem", Management Science, vol. 6, pp. 80-91, 1959.

[2] R.J. Kuo, F.E. Zulvia, and K. Suryadi,'Hybrid particle swarm optimization with genetic algorithm for solving capacitated vehicle routing problem with fuzzy demand - A case study on garbage collection system ".Applied Mathematics and Computation, vol. 219, no. 5, pp. 2574-2588, November 2012.

[3] H. Nazif, and L.S. Lee, “Optimized, ” Applied Mathematical Modeling, vol. 36, no. 5, pp. 2110-2117, May 2012.

[4] D. Ali, K. Devika, and M. Kaliyan, "Davor Svetinovic. An optimization model for product returns using genetic algorithms and artificial immune system", Resources, Conservation and Recycling, vol. 74, pp. 156-169, May 2013

[5] S. Karakatič, and V. Podgorelec, "A survey of genetic algorithms for solving multi depot vehicle routing problem," Applied Soft Computing, In Press, Uncorrected Proof, Available online 21 November 2014

[6] C.J. Ting, and C.H. Chen, "A multiple ant colony optimization algorithm for the capacitated location routing problem," International Journal of Production Economics, vol. 141, no. 1, pp. 34-44, January 2013.
[7] J. Tang, Y. Ma, J. Guan, and C. Yan, "A Max-Min Ant System for the split delivery weighted vehicle routing problem," Expert Systems with Applications, vol. 40, no. 18, pp. 7468-7477, December 2013.

[8] Y. Marinakis, and M. Marinaki, "A hybrid genetic - Particle Swarm Optimization Algorithm for the vehicle routing problem," Expert Systems with Applications, vol. 37, no. 2, pp. 1446-1455, March 2010

[9] Y. Marinakis, M. Marinaki, and G. Dounias, "A hybrid particle swarm optimization algorithm for the vehicle routing problem," Engineering Applications of Artificial Intelligence, vol. 23, no. 4, pp. 463-472, June 2010

[10] F. Carlos, and P.J. Fleming, "Multiobjective Optimization and Multiple Constraint Handling with Evolutionary Algorithms-Part 1:A Unifed Formulation," IEEE Transactions on Systems, Man and Cybernetics-Part A: Systems and Humans, vol. 28, no. 1, pp. 26$37,1998$.

[11] Index of SYMPHONY, Information on http://www.branchandcut. org/

[12] S.W. Lin, Z.J. Lee, K.C. Ying, and C.Y. Lee, "Applying hybrid meta-heuristics for capacitated vehicle routing problem," Expert Systems with Applications, vol. 36, pp. 1505-1512, 2009.

(C) LinHui and Luo; Licensee Bentham Open.

This is an open access article licensed under the terms of the Creative Commons Attribution Non-Commercial License (http://creativecommons.org/licenses/by-nc/3.0/) which permits unrestricted, non-commercial use, distribution and reproduction in any medium, provided the work is properly cited. 